\title{
Effect of oral administration of Propionibacterium acnes on growth performance, DTH response and anti-OVA titers in goat kids ${ }^{1}$
}

\author{
Luis Miguel Ferrer ${ }^{2}$, Antonio Fernández ${ }^{2 *}$, Araceli Loste ${ }^{2}$, Aurora Ortín², Delia Lacasta ${ }^{2}$, \\ Juan José Ramos ${ }^{2}$, María Teresa Verde ${ }^{2}$ and Tomás Conde ${ }^{2}$
}

\begin{abstract}
Ferrer L.M., Fernández A., Loste A., Ortín A., Lacasta D., Ramos J.J., Verde M.T. \& Conde T. 2013. Effect of oral administration of Propionibacterium acnes on growth performance, DTH response and anti-OVA titers in goat kids. Pesquisa Veterinária Brasileira 33(1):5-10. Departamento de Patología Animal, Facultad de Veterinaria, Universidad de Zaragoza, Miguel Servet 177, 50013-Zaragoza, Spain. E-mail: afmedica@unizar.es

Immunostimulants are susbstances that stimuli the response of effector cells to activate the immune response such as antigen uptake, cytokine release or antibody response. These substances can increase resistence to infection by different types of microorganisms, reducing dependence of antibiotics used in livestock animals. Recent reports have demonstrated the positive effect of Propionibacterium acnes (P. acnes) to control animal diseases. In this study, we evaluated the effect of the non-specific immunostimulant $P$. acnes on immunological functions and growth performance in goat kids. Twenty five goat kids served as control group (A) and another 25 animals received P. acnes being the experimental group (B). Kids were challenged with ovalbumin (OVA) to assess humoral immunity. To assess in vivo cell immunity, delayed type hypersensitivity (DTH) test with phytohemagglutinin (PHA) was used, clinical signs and body weight were recorded each week until 9 weeks of age when the experiment ended. Blood samples were obtained to analyze serum proteins fractions and anti-OVA specific antibodies. No clinical signs of disease and no differences ( $p>0.05$ ) on body weight between groups were recorded $(7.32 \pm 0.81 \mathrm{~kg}$ in group A, $7.13 \pm 0.65 \mathrm{~kg}$ in group B). Goat kids from group B had more total protein $(59.8 \pm 5 \mathrm{~g} / \mathrm{l})$ and albumin levels $(32.8 \pm 3.3 \mathrm{~g} / \mathrm{l})$ than goat kids from group A $(56.6 \pm 5.7 \mathrm{~g} / \mathrm{l}, 29.6 \pm 3.9 \mathrm{~g} / \mathrm{l}$ respectively) $(\mathrm{p}<0.05)$. DTH response in goat kids from group B on day 42 was higher $(\mathrm{p}<0.05)$ than group A. At day 63, goat kids from group receiving $P$. acnes had higher percentage (85.4) of anti-OVA IgM titers ( $\mathrm{p}<0.05$ ) than control group (57.7). In conclusion, the results showed that oral administration of $P$. acnes to goat kids improved some aspects of the immune system of the animals and it could be used to control goat diseases.
\end{abstract}

INDEX TERMS: Propionibacterium acnes, OVA, immunostimulant, goat kids, immunitary response.

\section{INTRODUCTION}

Ruminant neonates are born agammaglobulinemics, thus they are especially susceptible to infectious diseases and colostrum immunoglobulin intake is necessary to reach the adequate passive immunity (Weaver et al. 2000, Tizard 2009). Strategies to improve the survival in neonates are based on the increase of the passive immunity giving more immunoglobulin or colostrum substitute (Besser \&

\footnotetext{
${ }^{1}$ Received on June 20, 2012.

Accepted for publication on September 20, 2012.

${ }^{2}$ Departamento de Patología Animal, Facultad de Veterinaria, Universidad de Zaragoza, Miguel Servet 177, 50013-Zaragoza, Spain. *Corresponding author: afmedica@unizar.es
}

Gay 1994, Constant et al. 1994, Johnson et al. 2007, Godden et al. 2008, Poulsen et al. 2010, Moretti et al. 2012). These substitutes are lyophilized bovine colostrum or bovine serum-based colostrum which are commercial available to use directly to animals. Also, pasteurization of colostrum have been used as strategy to control transmition Maedi-Visna virus to newborn (Fernández et al. 2006, Loste et al. 2008), or destruction of Mycobacterium paratuberculosis experimental inoculated in the cow's colostrum (Stabel et al. 2004). The disadvantage of this method is that heat treatment of the colostrum resulted in a reduction in colostral IgG between 25-30\% (Stabel et al. 2004, Fernández et al. 2006), but without affect animal's health.

Other possibility is to use an immunostimulant in or- 
der to increase the active immunity. Immunostimulants are products that induce non antigen enhancement of innate or adaptative immunity (Ryan et al. 2010) and tend to stimulate immunity with minimal or no tissue damage (Mutwiri et al. 2007). The bests immunomodulators that could be used in veterinary medicine are cytokines, but they are very expensive, so another possibility is to induce cytokine regulatory cascade with pharmaceutical products, microbial agents, and mutraceuticals (Blecha 2001, Tizard 2009). In this way, the administration of non-specific immunostimulants has been used in horses (Flaminio et al. 1998, Rush 2006, Ryan et al. 2010) for treatment of respiratory diseases and at length as adjuvant to veterinary vaccines $(\mathrm{Mu}-$ twiri et al. 2007). The mechanism of action of non-specific immunostimulant is the activation of macrophages which subsequently activate terminal immune response such as antigen uptake, cytotoxicity, phagocytosis, cytokine release and antibody production (Flaminio et al. 1998, Rush 2006, Tizard 2009). Propionibacterium acnes, formerly known as Corynebacterium parvum, is a non-spore Gram positive bacteria and is considered an opportunistic pathogens (Perry \& Lambert 2006). P. acnes is a potent stimulator of the reticuloendothelial system (RES) (Megid \& Kaneno 2000, Megid et al. 2004, Tizard 2009) that enhanced both macrophage and lymphocyte function, natural killer cytotoxicity and cytokine production in laboratory animals. It has been recognised that $P$. acnes contribute the induction of pro-inflammatory cytokines IL- $1 \alpha$, IL-1 $\beta$, IL-8 and TNF- $\alpha$ (Perry \& Lambert 2006) and increase IL-10 in mice infected with street rabies virus (Megid et al. 2004). P. acnes is able to modulate the late phase reaction of the type I hypersensitivity in rodents and it has capacity to potentiate or suppress the Th2 response to OVA challenge, depending on the treatment protocol used (Braga et al. 2003). In this direction, Squaiella et al. (2008) demonstrated that purified soluble polysaccharide of $P$. acnes is the responsible the effects of modulation of immediate hypersensitivity reaction in mice.

Many of the experiments have been performed with laboratory animals and little is known about the effect of $P$. acnes in livestock. We have not found any reference about the immunological activity of $P$. acnes on the systemic immune response in caprine livestock. The objectives of the present work was to evaluate the effect of the oral administration of $P$. acnes in goat kids of 6 days of age on growth performance, humoral immunity after challenged with OVA, and determination of the in vivo cellular immunity to PHA by a DTH test.

\section{MATERIALS AND METHODS}

\section{Animals and feeding management}

The Ethics Committee of the University of Zaragoza approved the experimental protocol of this study. One hundred Murciano-Granadina goats of a commercial milk producer farm in Zaragoza (Spain) were synchronized with $45 \mathrm{mg}$ of fluorogestone acetate intravaginal sponges. After removal of the sponges, $500 \mathrm{IU}$ of PMSG (Pregnant Mare's Serum Gonadotropin) was injected to synchronize multiple births. Ultrasonography examination of the pregnancy was performed and fifty goats were chosen. All births were twin and 50 female kids were eligible for the study. The kids were immediately separated from their dams, their umbilical cords disinfected and injected with a $2 \mathrm{ml}$ intramuscular vitamin $\mathrm{AD}_{3} \mathrm{E}$ dose (Biosvita $\mathrm{AD}_{3} \mathrm{E}$ parenteral, Lab. Ovejero, León, Spain). Goat kids were fed with $160 \mathrm{ml} / \mathrm{kg}$ body weight pooled natural colostrum obtained from goats of the same farm a few weeks before. The total amount of colostrum was divided into three equal parts and given at 3,10 and 17 hours after birth. All kids included in this study drank all colostrum administered by nipple bottle. When the kids of both groups were 24 hours old, they were fed with artificial powdered milk, reconstituted in hot water $\left(40^{\circ} \mathrm{C}\right)$, three times a day using nipple bottles. At 12 days of age, a starter feed and barley straw were introduced.

The body weight was recorded at birth and at the end of each week until 9 weeks of age and the average daily gain (ADG) was calculated. To assess the clinical status, goat kids were observed twice daily to find of any clinical signs of disease.

\section{Administration of the immunostimulant}

Twenty five goat kids were used as control group without immunostimulant (group A), and $2 \mathrm{ml}$ of sterile saline was administered as a placebo. A suspension of formalin-killed $P$. acnes was used as immunostimulant. This was suspended in sterile saline at a concentration of $0.17 \mathrm{mg}$ dry bacteria per $\mathrm{ml}$, and given to 25 goat kids per os $(2 \mathrm{ml})$ mixed with artificial milk, twice a day during five days from 6 to 10 days of age (group B).

\section{Immunization}

All goat kids were subcutaneously immunized three times with ovalbumin (OVA) (Grade V, Sigma, USA). Injection was prepared with $300 \mu \mathrm{g}$ of OVA $/ \mathrm{ml}$ PBS and emulsified in $0.5 \mathrm{ml}$ Freund's incomplete adjuvant. Animals received a $1.5 \mathrm{ml}$ subcutaneous injection at 1, 4 and 7 weeks of the experiment.

\section{Blood collection and haematological analysis}

Blood samples ( $4 \mathrm{ml}$ ) were collected from the jugular vein and were added to a tube without anticoagulant to obtain serum. Samples were obtained at 2, 5 and 9 weeks of the experimental period. Blood obtained was allowed to clot and the serum was separated by centrifugation $\left(2000 \mathrm{xg}\right.$, at $4^{\circ} \mathrm{C}, 10$ minutes). The serum was frozen and stored at $-20^{\circ} \mathrm{C}$ until analysis.

\section{Protein serum electrophoresis}

Serum protein fractions were segregated by electrophoresis with a Hydrogel 7 kit (Sebia, France) and were read with a Shimadzu CS-9000 photodensitometer (Kyoto, Japan). Albumin, alpha-, beta- and gammaglobulins were calculated. Serum total protein was analysed with an $\mathrm{ACE}^{\circledR}$ autoanalyser (nํㅡㄹ AE5-23, Clinical Chemistry System, AlfaWassermann, USA) using the manufacturer's reagent and recommended procedures.

\section{Anti-OVA ELISA assay}

Anti-OVA immunoglobulins titres were measured using a non-competitive indirect ELISA, following the Kavanagh et al. (2003) method. Briefly, ovalbumin, immunoglobulins, and specific IgG, IgA and IgM antibodies in serum samples were detected by ELISA. Ninety-six-well ELISA plates (Microtiter, Labsystem, Finland) were coated overnight with ovalbumin by adding $100 \mu \mathrm{l}$ per well of a solution of $2.5 \mu \mathrm{g} / \mathrm{ml}$ of ovalbumin in sodium carbonate/bicarbonate buffer ( $\mathrm{pH} 9.6)$ at $4^{\circ} \mathrm{C}$ and then washed four times with washing fluid B (WFB, pH 7.2) containing 0.5\% Tween 20 and $1.85 \mathrm{~g}$ EDTA bi Na. One hundred microlitres per well of Block Ace (Serotec Ltd. UK) at 1/4 dilution was added and incubated for 30 minutes at $37^{\circ} \mathrm{C}$.

For anti-immunoglobulins-OVA serum samples were diluted $1 / 15000$ with Block Ace at 1/10 dilution. One hundred micro- 
litres of each sample was added in triplicate to the wells of the OVA-coated plates. The plates were incubated for 2 hours at room temperature, and washed thoroughly as before. One hundred microlitres of the secondary antibody (Rabbit anti-sheep/goat immunoglobulins conjugated with horse-radish peroxidase (HRP), (Serotec Ltd. UK) diluted 1/8000 in PBS were added to each well and incubated for 75 minutes at room temperature, following by washing as before. One hundred and sixty microlitres of the enzyme's substrate 3,3',5,5'-tetramethylbenzidine (TMB) (Chemicon International Ltd., UK) were added to each well and the colour development was stopped after 10 minutes by addition of $160 \mu \mathrm{l}$ of $\mathrm{H}_{2} \mathrm{SO}_{4} 2 \mathrm{M}$. Optical density (OD) values were read at $450 \mathrm{~nm}$ using an ELISA reader (Labsystem Multiskan RC, Finland) and means of the triplicate determination calculated. Also, anti IgG-OVA, anti IgM-OVA and anti IgA-OVA were determined, and the analysis were essentially the same with the following exceptions: for anti-IgG-OVA, the serum samples were diluted 1/20000 with Block Ace at 1/10 dilution. A donkey anti-sheep/goat IgG-HRP diluted $1 / 8000$ in PBS was used as secondary antibody. For anti IgA-OVA analysis, control and problem serums were diluted 1/10, and the secondary antibody (Donkey anti-sheep/goat IgA, Serotec Ltd, UK) $1 / 100$, and this was detected using a polyclonal rabbit anti-sheep immunoglobulins-HPR, (Serotec Ltd. UK) diluted to $1 / 2500$ in PBS. In the next steps, period of incubation and washing of the plates were done as before. For anti IgM-OVA, control and problem serums were diluted $1 / 10$ and the secondary antibody (Mouse anti-sheep/goat IgM, Serotec Ltd. UK) was diluted $1 / 1000$. Also, for anti IgM-OVA analysis, $100 \mu \mathrm{l}$ of tertiary antibody marked with HPR (polyclonal rabbit anti-sheep immunoglobulins-HPR, Serotec Ltd. UK) diluted to 1/10000 were added. In both cases, the enzyme substrate TMB was then added as described above.

The OD values for the total immunoglobulins, IgG, IgM and IgA ELISA were expressed as a ratio of a positive control sample present on each plate.

\section{Delayed type hypersensitivity test (DTH)}

DTH test was performed to assess in vivo cellular immunity. Each kid was injected intradermically on days 28 and 42 with $0.1 \mathrm{ml}$ of $2.5 \mathrm{mg} / \mathrm{ml}$ phytohemagglutinin (PHA, Sigma, USA.) in isotonic saline solution, at the central part of the costal area. Approximately $10 \mathrm{~cm}$ from this site, $0.1 \mathrm{ml}$ of saline solution was injected, serving as a control. Double skinfold thickness was measured using a constant tension calliper, immediately and 3, 6, 12, 24 and 48 hours after injection. Data were expressed as a ratio of skin thickness of PHA-injected to that of saline solution.

\section{Statistical analysis}

Results were analysed with a StatView 4.1 for Windows software. Data are reported as mean \pm SD. A non-parametric Mann-Whitney $U$ rank sum test was used for comparisons between groups. $\mathrm{p}$ value less than 0.05 were considered statistically significant.

\section{RESULTS}

Growth performance. No effect of the saline solution used as a placebo in the goats kids from group A was observed in any analysis developed in this investigation. No statistically significant differences ( $p>0.05)$ between groups were observed during the experiment when data of body weight were analyzed. At day 0 , mean body weight was $2.44 \pm 0.35$ $\mathrm{kg}$ in group A (range $1.80-3.20 \mathrm{~kg}$ ) and $2.56 \pm 0.33 \mathrm{~kg}$ in group B (range $1.60-3.20 \mathrm{~kg}$ ). At the end of the study, the mean body weight recorded in group A was $7.32 \pm 0.81 \mathrm{~kg}$ (range 6.10-8.80 kg) and in group B was $7.13 \pm 0.65 \mathrm{~kg}$ (range $6.00-8.50 \mathrm{~kg})$. The ADG at the end of 9 weeks were 77.46 g/day in group A (range 55-105.3g/day) and $72.47 \mathrm{~g} /$ day in group B (range 60-96.6g/day) and no statistically significant differences ( $p>0.05)$ was noted. Goat kids in both groups appeared to be clinically normal and no deaths were recorded.

Protein serum electrophoresis. Statistical analysis of the data showed a difference $(\mathrm{p}<0.05)$ on TP $(56.6 \pm 5.7 \mathrm{~g} / \mathrm{l}$ in group $A, 59.8 \pm 5 \mathrm{~g} / \mathrm{l}$ in group $\mathrm{B}$ ) and albumin concentration $(29.6 \pm 3.9 \mathrm{~g} / \mathrm{l}$ in group $\mathrm{A}, 32.8 \pm 3.3 \mathrm{~g} / \mathrm{l}$ in group $\mathrm{B})$ at day 14 , and performed a higher $A / G$ ratio in this group $(1.22 \pm 0.13 ; 1.11 \pm 0.15$ in group $A ; p<0.001)$. At day 35 , albumin concentration was still higher in the group that received the immunostimulant $(34.3 \pm 7.2 \mathrm{~g} / \mathrm{l}, 30.7 \pm 6.5 \mathrm{~g} / \mathrm{l}$ in group $A ; p<0.05)$. At the end of the experiment, globulin levels in group B was lower $(21.7 \pm 4.5 \mathrm{~g} / \mathrm{l})$ when data were compared with group A $(24.2 \pm 3.9 \mathrm{~g} / \mathrm{l}, \mathrm{p}<0.05)$ and $\mathrm{A} / \mathrm{G}$ ratio was higher $(1.55 \pm 0.31$ and $1.41 \pm 0.23$ in group A; $\mathrm{p}<0.05$ ).

DTH test. Table 1 shows results of DTH test. At day 28 , no statistically significant differences $(p>0.05)$ were found between groups in DTH test. When goat kids were 42 days old, statistically significant differences were observed for the response to PHA injection between the group receiving the immunostimulant (higher response) and the control group (lower response) at 3, 6, 12 and 48 hours.

Table 1. Delayed type hypersensitivity test (DTH) of goat kids from control group (A) and receiving an immunostimulant (group B)

\begin{tabular}{cccccc}
\hline Hour & \multicolumn{2}{c}{ Day 28 } & & \multicolumn{2}{c}{ Day 42 } \\
\cline { 2 - 3 } \cline { 5 - 6 } & Group A & Group B & & Group A & Group B \\
\hline 0 & $0.98 \pm 0.18$ & $0.99 \pm 0.15$ & & $1.05 \pm 0.23$ & $1.05 \pm 0.21$ \\
3 & $1.45 \pm 0.39$ & $1.41 \pm 0.39$ & & $1.18 \pm 0.19$ & $1.28 \pm 0.14^{*}$ \\
6 & $1.30 \pm 0.34$ & $1.32 \pm 0.32$ & & $1.12 \pm 0.14$ & $1.21 \pm 0.14^{*}$ \\
12 & $1.44 \pm 0.35$ & $1.38 \pm 0.38$ & & $1.45 \pm 0.51$ & $1.77 \pm 0.49^{*}$ \\
24 & $1.33 \pm 0.27$ & $1.29 \pm 0.25$ & & $1.32 \pm 0.53$ & $1.49 \pm 0.38$ \\
48 & $1.20 \pm 0.21$ & $1.21 \pm 0.24$ & & $1.19 \pm 0.25$ & $1.39 \pm 0.33^{*}$
\end{tabular}

Data are mean \pm SD, $(n=25) .{ }^{*} \mathrm{p}<0.05$, comparing groups $A$ and $B$ at each time point. Data is expressed as ratio between thickness of the skin with PHA and isotonic saline solution.

Antibody response to OVA. Antibody response following OVA inoculation is shown in Figure 1. The percentage was increasing by time, but there were no statistically significant differences ( $\mathrm{p}>0.05$ ) between groups in total immunoglobulins and IgG levels when data were analyzed during the experimental period. However, IgM levels were increased by time and at day 63 , a high percentage was found in the group receiving the immunostimulant $(\mathrm{p}<0.05)$. OVA-specific IgA concentrations were very low in both groups of animals (data not show).

\section{DISCUSSION}

The present study investigated the influence of Propionibacterium acnes on the growth, humoral and cell-mediated immune response in goat kids. In this experiment, no mor- 

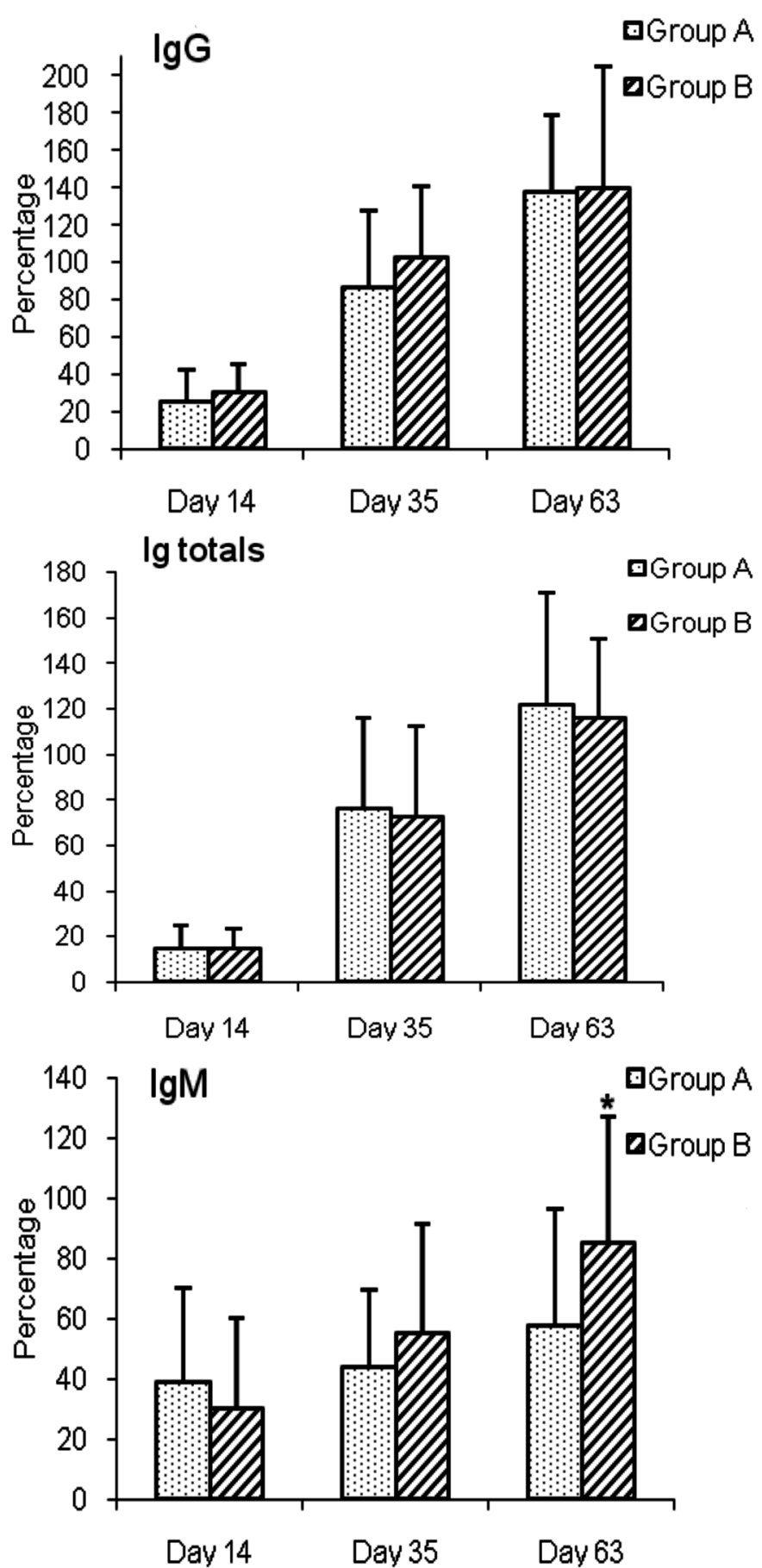

Fig. 1. Serum OVA-specific antibodies from goat kids of control group (A) and receiving an immunostimulant (group B). *(superscript lowercase letter) indicate statistically significant differences between the two groups $(p<0.05)$. Please note the different scales of the vertical axes.

tality or clinical signs of disease of any kids of both groups were recorded. Also, application of the immunostimulant had no effect on body weight and ADG. There is not any reference of $P$. acnes effect on growth performance in the livestock. The use of an immunomodulator such as inactivated Parapoxvirus improved significantly growth performance in piglets compared with controls (Kyriakis et al. 1998) and was due that immunomodulator could be effec- tive against postweaning diarrhoea syndrome and wasting pig syndrome. In our experiment, in the goat kids no diseases or death were observed, so no effect of the immunostimulant on health could be observed. Neither, no adverse effect of $P$. acnes on health parameters were recorded. Flaminio et al. (1998) reported a high rectal temperature on foals after injection of P. acnes, and no effects were observed either in foals whose mothers had been inoculated before foaling with $P$. acnes to increase the quality of the mare's colostrum (Turner et al. 2003). Recently, Ryan et al. (2010) did not record any adverse effect of an inactivated P. acnes administrated intravenously in newborn foals and these animals had less intracellular proliferation of Rhodococcus equi within macrophages. However, Corrier \& Ziprin (1989) observed a significant increase of morbidity in one-day-old broiler chickens inoculated with C. parvum and challenged with Salmonella typhimurium. For these authors, the data recorded may reflect differences in the modulators effects of the immunostimulant in chickens. Immunostimulants can produce systemic reactions such as fever, malaise, diarrhoea, hypotension or local inflammation following its administration and these effects are associated with cytokine release by macrophages (Rush 2006, Tizard 2009). The intensity of the effects depends of the multiple dose of the immunostimulant given. Inthis experiment, the immunostimulant was administered per os, not by parenteral injection as it has been used in other studies (Corrier \& Ziprin 1989, Flaminio et al. 1998, Turner et al. 2003, Ryan et al. 2010). Probably, this via of administration did not put in contact sufficiently the killed bacteria with the macrophages and this could explain lack of secondary effects found in our experiment.

In this study, the segregation of serum proteins shows normal values for all fractions and slightly higher values for albumin concentrations (normal values 26-31.7g/l) found in our laboratory for Murciano-Granadina goat kids (Fernández et al. 2006) mainly on day 14 in the group B. The results were similar to those found in a previous study (Fernández et al. 2006, Loste et al. 2008). The ingestion of high quality colostrum during the first hours of life is essential for the synthesis and maintenance of the level of serum proteins (Besser \& Gay 1994, Constant et al. 1994, Weaver et al. 2000). In this experiment, also the animals received sufficient quantity and quality of colostrum since the first days of life. One of the reasons for the increase in albumin level is the dehydration (Kaneko 2008), but this condition was not observed in any of the goat kids included in our study. No effects of the P. acnes have been previously reported on the albumin synthesis and no explanation could be found to explain these results. Also, the increase in $A / G$ ratio observed only can be explained due to increase in albumin level.

The intradermal injection of PHA produces a local tissue reaction, stimulating $\mathrm{T}$ lymphocytes similar to that DTH, being a rapid method to assess the cellular immunity in vivo (Tizard 2009) and has been successfully used in our laboratory in both goat kids and lambs (Fernández et al. 2006, Loste et al. 2008). Cell immunity is an effector function of lymphocyte and the results of DTH test indicate 
that goat kids treated with an immunostimulant enhanced cell-mediated immunity (CMI). The lack of DTH response in goat kids on day 28 could result from an insufficient antigen stimulus for induction of high levels of DTH. Also, this difference may be due to the experimental protocol used in our experiment. Braga et al. (2003) observed that type of protocol administrating $P$. acnes influenced in the outcome of the immune response in mice because when HEW subcutaneous implant was made one week after administration of the immunomodulator, a typical DTH and cytokine Th1 response was recorded. This results demonstrated that $P$. acnes promoted or suppressed the CMI response depending on the treatment protocol (Squaiella et al. 2008). The effect of $P$. acnes on DTH response is contradictory and many reports describe an immunosuppressive activity. In this sense, Gauthier-Rahman (1981) found an immunoinhibitory effect of $C$. parvum on DTH in two strains of guinea pigs when animals were treated with cyclophosphamide. For Corrier \& Ziprin (1989) C. parvum suppressed significantly the DTH response to PHA in 1 day old broiler chickens, indicating that T-lymphocyte activity was suppressed. However, for Bomford (1980), C. parvum was the only adjuvant which consistently boosts DTH response to sheep blood cells in a mouse model.

In this experiment, the effect of the administration of an immunostimulant on humoral response was variable. Serum OVA-specific antibodies were increased by the time and at day 63 a higher value of IgM was found. Immunization with OVA produced a high and sustained systemic antibody response on immunoglobulins, meanly on IgG levels such as it has been observed in other species (Sheldrake et al. 1985, HogenEsch et al. 1996, Bowersock et al. 1998, Patel et al. 2007). The effect of an immunostimulant on antibody production depends on many factors such as the route of administration, timing of treatment in relation to antigen and the dose of the immunostimulant or the antigen. We administered the immunostimulant during five days when goat kids were six days old and a poor humoral immunitary response was observed. Perhaps, the oral administration was not the most appropriate route to cause a sufficient stimulus to promote to the effectory cells to produce antibodies. Neither Flaminio et al. (1998) did not find variations on serum IgG and IgM concentrations on foals injected with $P$. acnes.

In the goat kids, the immunostimulant was able to increase the serum levels of anti-OVA IgM at the end of the experiment. This could be due to the fact that $P$. acnes is a powerful stimulant of macrophages. These leukocytes phagocyte to the microorganisms and stimulate the cytokine synthesis; then, the macrophages stimulated enhance the IL-6 production helping the maturing process of the B lymphocytes in plasmatic cells. IL- 6 together with IL-1 is an important cofactor in the synthesis of IgM (Tizard 2009). Very little anti-OVA IgA specific antibody was presented in both groups of goat kids when OVA was injected subcutaneously. Similar result was reported by HogenEsch et al. (1996) who did not find anti-OVA IgA in the serum of calves challenged with OVA, but high levels could detect in bronchoalveolar lavage. On the contrary, when OVA were administrated by intranasal, intraperitoneal or intrabronchial route (Bowersock et al. 1998, Kavanagh et al. 2003, Patel et al. 2007), vaginal, nasal fluids and faeces had high levels of IgA. These results suggest that OVA subcutaneous immunization primer for an IgG and IgM production, but not for a mucosal IgA response. The IgA is meanly secreted by intestinal mucosa and has its function in the protection of mucosal surface neutralising microorganisms both extracellularly in mucosal secretions and intracellularly following infection of epithelial cells at mucosal surfaces (Kavanagh et al. 2003). Furthermore, the ruminants have less IgA than other immunoglobulins (Tizard 2009).

\section{CONCLUSION}

The results of the present study indicate that administration of the non-specific immunostimulant Propionibacterium acnes enhanced both cellular and humoral immunity demonstrated by increasing DTH response to PHA and levels of anti-OVA IgM in serum. The immunostimulant administration could be used to improve immunological status on the first month of life. However, more investigations are needed to determine the optimum doses and route of administration to improve the immunitary response of the goat kids.

Acknowledgements.- This research was funded by a grant from Diputación General de Aragón (group A30, Patología de la Nutrición en Pequeños Rumiantes).

\section{REFERENCES}

Besser T.E. \& Gay C.C. 1994. The importance of colostrum to the health of the neonatal calf. Vet. Clin. North Am., Food Anim. Pract. 10:107-117.

Blecha F. 2001. Immunomodulators for prevention and treatment of infectious disease in food-producing animals. Vet. Clin. North Am., Food Anim. Pract. 17(3):621-633.

Bomford R. 1980. The comparative selectivity of adjuvants for humoral and cell-mediated immunity. II. Effect on delayed-type hypersensitivity in the mouse and guinea pigs, and cell-mediated immunity to tumour antigens in the mouse of Freund's incomplete and complete adjuvants, alhydrogel, Corynebacterium parvum, Bordetella pertussi, muralmyl dipeptide and saponin. Clin. Exp. Immunol. 39:435-441.

Bowersock T.L., HogenEsch H., Torregrosa S., Borie D., Wang B., Park H. \& Park K. 1998. Induction of pulmonary immunity in cattle by oral administration of ovalbumin in alginate microspheres. Immunol. Lett. 60:37-43.

Braga E.G., Ananias R.Z., Mussalem J.S., Squaiella C.C., Longhini A.L.F., Mariano M., Travassos L.R. \& Longo-Maugéri I.M. 2003. Treatment with Propionibacterium acnes modulates the late phase reaction of immediate hypersensitivity in mice. Immunol. Lett. 88:163-169.

Constant S.B., Leblanc M.M., Klapstein E.F., Beebe D.E., Leneau H.M. \& Nunier C.J. 1994. Serum immunoglobulin G concentration in goat kids fed colostrum or a colostrum substitute. J. Am. Vet. Med. Assoc. 205:17591762.

Corrier D. \& Ziprin R.L. 1989. Suppression of resistance to Salmonella typhimurium in young chickens inoculated with Corynebacterium parvum. Avian Dis. 33:787-791.

Fernández A., Ramos J.J., Loste A., Ferrer L.M., Figueras L., Verde M.T. \& Marca M.C. 2006. Influence of colostrum treated by heat on immunity function in goat kids. Comp. Immunol. Microbiol. Infect. Dis. 29:353364.

Flaminio M.J.B., Rush B.R. \& Shuman W. 1998. Immunologic function in horses after non-specific immunostimulant administration. Vet. Immunol. Immunopathol. 63: 303-315.

Gauthier-Rahman S. 1981. Immunomodulation by Corynebacterium par- 
vum in two strains of guinea-pigs and the effect of cyclophosphamide. Immunol. 42:99-109.

Godden S.M., Haines D.M. \& Hagman D. 2008. Improving passive transfer of immunoglobulins in calves. I. Dose effect of feeding a commercial colostrum replacer. J. Dairy Sci. 92:1750-1757.

HogenEsch H., Torregrosa S., Borie D., Gaskell C. \& Bowersock T.L. 1996. Systemic and pulmonary immune response to intrabronchial administration of ovalbumin in calves. Vet. Immunol. Immunopathol. 51:293-302.

Johnson J.L., Godden S.M., Molitor T., Ames T. \& Hagman D. 2007. Effects of feeding heat-treated colostrum on passive transfer of immune and nutritional parameters in neonatal dairy calves. J. Dairy Sci. 90:5189-5198.

Kaneko J.J. 2008. Proteins, proteomics, and the dysproteinemias, p.117155. In: Kaneko J.J., Harvey J.W. \& Bruss M.L. (Eds), Clinical Biochemistry of Domestic Animals. $6^{\text {th }}$ ed. Academic Press, San Diego.

Kavanagh O.W., Earley B., Murray M., Foster C.J. \& Adair B.M. 2003. Antigen-specific IgA and IgG responses in calves inoculated intranasally with ovalbumin encapsulated in poly(DL-lactide-co-glycolide) microspheres. Vaccine 21:4472-4480.

Kyriakis S.C., Tzika E.D., Lyras D.N., Tsinas A.C., Saoulidis K. \& Sarris K. 1998. Effect of an inactivated Parapoxvirus based immunomodulator (Baypamun) on post weaning diarrhoea syndrome and wasting pig syndrome of piglets. Res. Vet. Sci. 64:187-190.

Loste A., Ramos J.J., Fernández A., Ferrer L.M., Lacasta D., Verde M.T., Marca M.C. \& Ortín A. 2008. Effect of colostrum treated by heat on immunological parameters in newborn lambs. Livest. Sci. 117:176-183.

Megid J. \& Kaneno R. 2000. Natural killer activity in mice infected with rabies virus submitted to $P$. acnes (Propionibacterium acnes) as immunomodulator. Comp. Immunol. Microbiol. Infect. Dis. 23: 91-97.

Megid J., Kaneno R., Nozaki C.N., Brito C.J.C. \& Almeida M.F. 2004. Increased interleukin-10 associated with low IL- 6 concentrations correlated with greater survival rates in mice infected by rabies virus vaccinated against it and immunomodulated with P. acnes. Comp. Immunol. Microbiol. Infect. Dis. 27:394-411.

Moretti D.B., Nordi W.M., Lima A.L., Pauletti P., Susin I. \& Machado-Neto R. 2012. Lyophilized bovine colostrum as a source of immunoglobulins and insulin-like growth factor for newborn goat kids. Livest. Sci. 145: 223-229.

Mutwiri G., Gerdts V., López M. \& Babiuk L.A. 2007. Innate immunity and new adjuvants. Rev. Sci. Tech. OIE 26:147-156.
Patel G.B., Zhou H., Ponce A. \& Chen W. 2007. Mucosal and systemic immune responses by intranasal immunization using archaeal lipid-adjuvanted vaccines. Vaccine 25:8622-8636.

Perry A.L. \& Lambert P.A. 2006. Propionibacterium acnes. Letters Appl. Microbiol. 42:185-188.

Poulsen K.P., Foley A.L., Collins M.T. \& McGuirck S.M. 2010. Comparison of passive transfer of immunity in neonatal dairy calves fed colostrum or bovine serum-based colostrum replacement and colostrum supplement products. J. Am. Vet. Med. Assoc. 15:949-954.

Rush B.R. 2006. Immunomodulatory therapy. Proc. North America Veterinary Conference (NAVC). Internet Publisher, International Veterinary Information Service: <http://www.ivis.org/proceedings/navc/2006/ LA/080.asp?LA=1> Accessed Dec. 5, 2011.

Ryan C., Giguère S., Fultz L., Long M.T. \& Crawford P.C. 2010. Effects of two commercial available immunostimulants on leukocyte function of foals following ex vivo exposure to Rhodococcus equi. Vet. Immunol. Immunopathol. 138:198-205.

Sheldrake R.F., Husband A.J., Watson D.L. \& Cripps A.W. 1985. The effect of intraperitoneal and intramammary immunization of sheep on the numbers of antibody-containing cells in the mammary gland and antibody titres in blood serum and mammary secretions. Immunol. 56:605-614.

Squaiella C.C., Longhini A.L.F., Braga E.G., Mussalem J.S., Ananias R.Z., Yendo T.M., Straus A.H., Toledo M.S., Takahashi H.K., Hirata I.Y. \& Longo-Maugéri I.M. 2008. Modulation of the type I hypersensitivity late phase reaction on OVA by Propionibacterium acnes-soluble polysaccharide. Immunol. Lett. 212:157:166.

Stabel J.R., Hurd S., Calvente L. \& Rosenbusch R.F. 2004. Destruction of Mycobacterium paratuberculosis, Salmonella spp., and Mycoplasma spp. in raw milk by a commercial on-farm high-temperature, short-time pasteurizer. J. Dairy Sci. 87:2177-2183.

Tizard I.R. 2009. Introducción a la Inmunología Veterinaria. $8^{a}$ ed. Elsevier Saunders, Barcelona, Spain.

Turner J.L., Arns M.J. \& Minton J.E. 2003. Case study: effects of non-specific immunostimulation of prepartum mares on colostral quality and foal immune function. Prof. Anim. Sci.19:62-67.

Weaver D.M., Tyler J.W., Vanmetre D.C., Hostetler D.E. \& Barrington G.M. 2000. Passive transfer of colostral immunoglobulins in calves. J. Vet. Intern. Med. 14:569-577. 